\title{
Effects of Antecedent Pruning in Fuzzy Classification Systems
}

\author{
Andreas Nürnberger, Aljoscha Klose, Rudolf Kruse \\ University of Magdeburg, Faculty of Computer Science \\ 39106 Magdeburg, Germany \\ E-mail: andreas.nuernberger@cs.uni-magdeburg.de \\ Fax: +49 39167 12018, Phone: +49 3916711358
}

\begin{abstract}
Fuzzy classification rules are widely considered a well-suited representation of classification knowledge, as they allow readable and interpretable rule bases. The goal of this paper is to discuss the shapes of the resulting classification borders under consideration of different types of fuzzy sets, rule bases and t-norms and thus which class distributions can be represented by such classification systems. We focus on discussing how antecedent pruning influences the classification behaviour of fuzzy classifiers. Our main goal is to give the potential user an insight into the classification behaviour of fuzzy classifiers. For this, mainly $2 D$ and $3 D$ visualisations are used to illustrate the cluster shapes and the borders between distinct classes.
\end{abstract}

\section{Introduction}

Fuzzy classification rules are widely considered a well-suited representation of classification knowledge, as they allow readable and interpretable fuzzy rule bases [2]. Due to their abstraction from numbers to linguistic variables they resemble the way, humans would possibly formulate their knowledge [6]. Everybody can easily be convinced that the world is inherently fuzzy and that crisp thresholds can almost never be justified. Therefore, the use of fuzzy rules seems quite intuitive. Specifying fuzzy sets may seem easier than the specification of crisp intervals. One may hope that the inference system will 'somehow' deal with the uncertainties. Especially, when rule bases are automatically or half-automatically created from example data, this can hold true. But finally, when the derived classifier is applied to data, usually a winner takes all principle is used to determine a single class to which the applied data is most similar. Therefore, there are always crisp borders between regions of different predicted classes.

In [10] we had discussed the shape of these borders for two overlapping classes in two- and threedimensional universes and we had given general hints concerning the shape of higher dimensional clusters. Furthermore, we had investigated different influences that should be considered, when a fuzzy rule base is created (manually or automatically). These are, for example, the t-norm and t-conorm used, the use of threshold membership values, the type of fuzzy sets used, and the number and complexity of fuzzy rules used to define the classifier.

In this paper we will discuss the effects of pruning methods under consideration of the aspects mentioned above. These effects are especially interesting in the context of neuro-fuzzy learning, since they give an insight into the operation of different learning techniques.

However, our main goal is to give the potential user an intuition how antecedent pruning influences the classification behaviour of fuzzy classifiers.

First, we describe the general structure of fuzzy classification systems, and how they can be derived and modified. In the subsequent sections, we use 2D and $3 \mathrm{D}$ visualisations to illustrate the shapes of borders between distinct classes and the effects that result from pruning.

\subsection{Fuzzy classification systems}

The basic idea of fuzzy classification systems [2] is to describe the supposed clusters - to which the respective classes are assigned - by vague cluster prototypes. These prototypes are defined by a number of fuzzy sets which circumscribe the cluster in each considered dimension of the universe. Thus, a specific cluster $i$ is defined by a fuzzy classification rule of the form:

$$
\begin{aligned}
& \text { if } \quad x_{1} \text { is } \mu_{i, 1} \text { and } x_{2} \text { is } \mu_{i, 2} \text { and } \ldots x_{n} \text { is } \mu_{i, n} \\
& \text { then pattern }\left(x_{1}, x_{2}, \ldots, x_{n}\right) \text { belongs to class } c_{k},
\end{aligned}
$$

where $\mu_{i, j}$ is a membership function that defines the fuzzy set describing the cluster $i$ in dimension $j$. (In the following we use for simplification the fuzzy sets $(A, B, \ldots)$ instead of their membership functions $\mu$ to define fuzzy rules). For the evaluation of a fuzzy (classification) rule a t-norm has to be defined, by which the activation of the rule and thus the membership value of the pattern to the specific class $c_{k}$ can be derived. Common t-norms used for fuzzy classification systems are minimum (min) and algebraic product (prod) [6]. If more than one rule predicts the same class usually the highest rule activation is chosen ( $\max$ t-conorm). 


\subsection{Obtaining fuzzy classifiers}

Conventional classifiers are usually derived from data and are not specified manually, due to their (mostly) complex mathematical description. In contrast to this, fuzzy classifiers can easily be defined manually and learnt classifiers can more easily be interpreted. The most common methods to induce fuzzy classifiers from data are fuzzy clustering and neuro-fuzzy learning.

Fuzzy clustering searches for clusters in the input data (see, for example, [1][2]). The number of clusters is determined by an evaluation measure, and the size and shape of the clusters is given by the clustering algorithm. The obtained clusters can be transformed into fuzzy rules by projection of the clusters onto the individual dimensions [4].

Neuro-fuzzy learning combines fuzzy classifiers with neural networks (see, for example, [7]). These approaches create fuzzy classifiers from data by heuristic learning procedures. Usually the neurofuzzy learning procedures underlie certain restrictions, such that both, acceptable performance and interpretable rules can be obtained [8].

\subsection{Pruning of fuzzy classifiers}

To get more interpretable fuzzy rule bases, that also generalise well on unseen data, it is often necessary to prune rule bases. There are generally two ways to simplify a rule base: The deletion of unnecessary rules and the simplification of individual rules.

The deletion can either be done manually - for example after testing plausibility or performance - or automatically. Automatic pruning in neuro-fuzzy classifiers can be seen as structural learning.

In this paper we focus on illustrating the effects of antecedent pruning, i.e. deleting single antecedents from rules. We restrict ourselves to triangular fuzzy sets. However, the observed effects are similar for any convex fuzzy set. For an analysis of fuzzy classifiers with arbitrary fuzzy sets see [3]. However, these fuzzy sets can hardly be interpreted in linguistic terms and are thus unusual in practice.

\section{Classifiers in a 2D universe}

Figure 1 depicts an example of a simple fuzzy classification system consisting of the three fuzzy rules

$R_{1}$ : If $x$ is $A_{1}$ and $y$ is $B_{1}$ then $(x, y)$ belongs to $c_{1}$ $R_{2}$ : If $x$ is $A_{2}$ and $y$ is $B_{2}$ then $(x, y)$ belongs to $c_{2}$ $R_{3}$ : If $x$ is $A_{3}$ and $y$ is $B_{3}$ then $(x, y)$ belongs to $c_{3}$, where the fuzzy sets $A_{i}$ and $B_{i}$ are defined by triangular membership functions. The pyramids $R_{1}, R_{2}$ and $R_{3}$ in Figure 1a represent the membership degrees of each data point $(x, y)$ to the respective classes $c_{i}$, obtained by use of the $\min$ t-norm. In Figure $1 \mathrm{~b}$ the resulting class borders and the contour lines of equal membership values are represented in a projection onto the $(x, y)$-plane. To derive the predicted class a winner takes all principle was used. Figure 1c represents the resulting class borders and contour lines if the t-norm prod is used (see also [10]).

To visualise the effect of antecedent pruning we removed the antecedent " $x$ is $A_{1}$ " from the first rule. So, we replace $R_{l}$ with:

$R_{1}^{\prime}$ : If $y$ is $B_{1}$ then $(x, y)$ belongs to $c_{1}$.

As depicted in Figure 2a, the pyramid defining the first class $c_{1}$ spreads out across the x-axis and takes over large parts which were assigned to class $c_{2}$ beforehand (cf. black hexagons in Figure 2). This occupation effect is even more severe if the t-norm prod is used (Figure 2b). Figure 2c shows the remaining area for class $c_{2}$ if $R_{3}$ is also pruned to

$R_{3}^{\prime}$ : If $x$ is $A_{3}$ then $(x, y)$ belongs to $c_{3}$.

The reason for the stronger impacts when using the algebraic product is that the activation of a rule $R$ evaluated by t-norm prod depends exponentially on the number of antecedents (see also [10]):

$$
\operatorname{prod}(R)=\prod_{i=1, \ldots, n} A_{i}\left(x_{i}\right)
$$

On the other hand, the occupation effect is minimal for the t-norm $\min$, since $\min$ is the greatest $\mathrm{t}$-norm (i.e. the greatest function that meets the definition of a t-norm).

This occupation effect is the reason for an usual

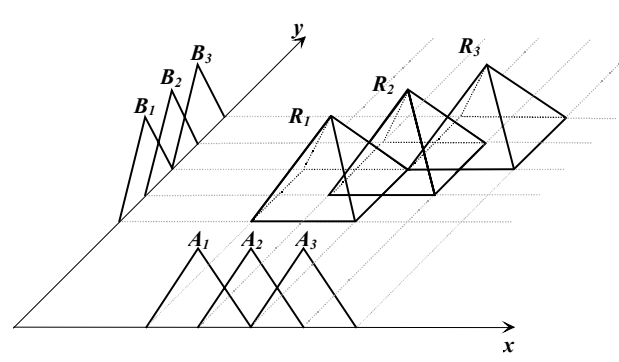

(a)

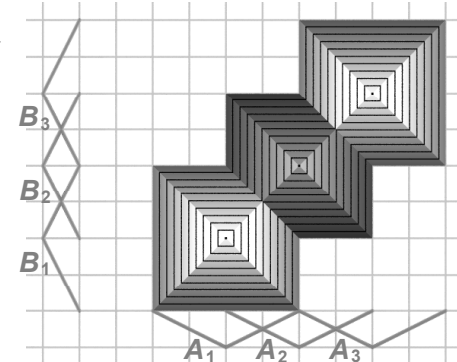

(b)

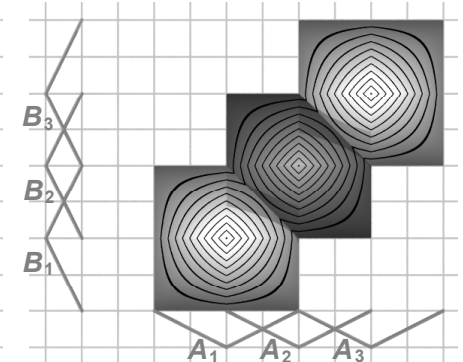

(c)

Figure 1. Representation of three fuzzy rules: Visualised membership degrees (a), projection of classes obtained by evaluation of a rule base with t-norm $\min$ (a) and t-norm prod (b) 
initial decrease in classification performance when a rule base has been pruned, since the pruned rule takes over correctly classified regions of the data space. The error which may result from a rule spreading out over one dimension can usually be neglected since - if the classifier has been optimised prior to pruning - these areas contain just very few data points. Often, the performance decrease can be compensated by an optimisation step after pruning [5]. During this learning step the 'reduced' fuzzy rule (e.g. $R_{2}$ in Figure 2) will be enlarged and shifted towards the 'grown' rule (here $R_{1}$ ).

However, this compensation requires adaptation of the fuzzy sets, ie. their support has to be enlarged. This may result in fuzzy sets that insufficiently represent the associated liguistic terms, and can therefore affect interpretability.

\section{Classifiers in a 3D universe}

In this section we consider fuzzy rules with antecedents in three dimensions, i.e. rules of the form:

$$
\begin{aligned}
\mathrm{R}_{\mathrm{i}} \text { : If } x \text { is } A_{\mathrm{i}} \text { and } y \text { is } B_{\mathrm{i}} \text { and } z \text { is } C_{i} \\
\text { then }(x, y, z) \text { belongs to } c_{i}
\end{aligned}
$$

To obtain usable diagrams of the resulting three dimensional fuzzy clusters we omit the (direct) representation of the membership degrees. Instead, we plot the outer cluster surfaces in the threedimensional universe. The plots are rotated for better visibility. However, the cluster borders are parallel to the axes of the coordinate system, since single clusters created by fuzzy rules are spanned by the fuzzy sets in the antecedents of the rule. Therefore, the axes of the spanning system are always parallel to the axes of the universe. Furthermore, for most of the commonly used t-norms holds $a>0 \wedge b>0 \quad T(a, b)>0$. So, the outer shapes of the clusters are usually defined by axes parallel hyper boxes.

Figure 3a shows a plot of two rules. The fuzzy sets add to 1, i.e. they overlap as the fuzzy sets in Figure 2. Figure $3 \mathrm{~b}$ shows the same rule base as Figure $3 \mathrm{a}$, but rotated and with one rule being hidden, so that the intersection surface between the classified areas can be examined. Figure 3e shows the same for tnorm prod instead of min.

Figures $3 \mathrm{c}$ and $3 \mathrm{f}$ illustrate what remains of the rule when one antecedent of the second rule is pruned (for min and prod, respectively). The outer shape of the hidden rule is a box enlarged to the bounds of the universe in the pruned dimension.

Figure $3 \mathrm{~d}$ and $3 \mathrm{~g}$ show the intersection surface when two antecedents are pruned from the hidden rule (again for min and prod). It can be seen, that the occupation effect is even more serious in the three dimensional case. Especially if two antecedents are removed from a rule, and if the generally less t-norm prod is used most of the overlapping area is taken over by the pruned rule.

To stress this, Figure $3 \mathrm{~h}$ shows the results of two hidden pruned rules. The rules might for example be:
$R_{1}$ : if $x$ is small
then $(x, y, z)$ belongs to $c_{1}$
$R_{2}$ : if $x$ is large
then $(x, y, z)$ belongs to $c_{1}$
$R_{3}$ : if $x$ is medium and $y$ is medium and $z$ is medium then $(x, y, z)$ belongs to $c_{2}$

We would expect that $R_{1}$ and $R_{2}$ influence the enclosed rule $R_{3}$. However, the size of the area occupied by rule $R_{3}$ is counter-intuitively small.

Fuzzy classifiers and probability theory. It is interesting to compare fuzzy classification rules and classifiers based on probability theory (e.g. naive bayes classifiers). In probability theory independent dimensions are combined using the product, which is also very common in fuzzy based classification. The most apparent difference is the normalisation of the probability densities to integrate to one in every dimension. Normalised fuzzy sets, however, are defined to have at least one point with a membership value of one, but may have any area below the curve. This leads to a considerably higher hyper volume under a rule if an antecedent is pruned, since all values of the corresponding attribute are considered as possible and therefore the membership degree is one for all possible values. The hyper volume

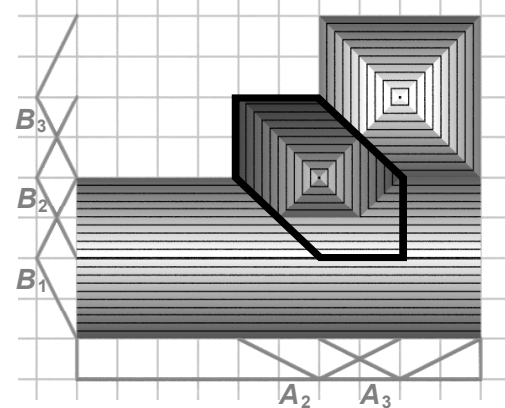

(a)

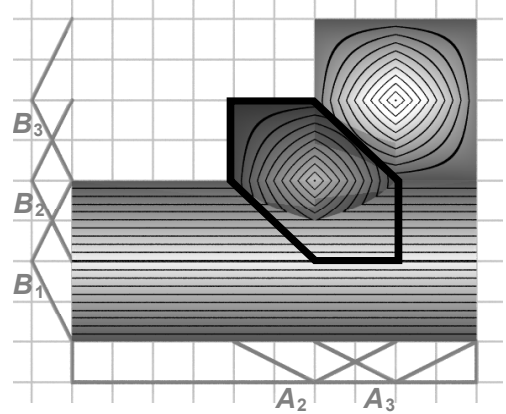

(b)

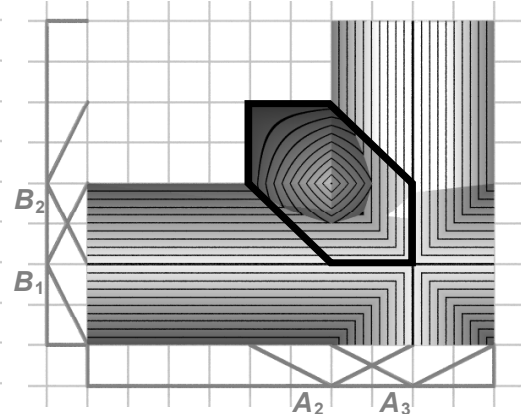

(c)

Figure 2. Pruned rule bases: Antecedent " $x$ is $A_{1}{ }^{\text {" }}$ removed and evaluated with t-norm $\min ($ a) and $\operatorname{prod}$ (b); Antecedents " $x$ is $A_{1}$ " and " $y$ is $B_{3}$ " removed and evaluated with t-norm prod (c) 
in the probabilistic case remains one by definition. For the pruned dimension we assume a uniform distribution. Thus, the density of a rule is decreased, when its influence spreads.

Nevertheless, naive bayes classifiers can be mapped to (neuro-)fuzzy classifiers, when rule weights are used [9]. In this case, the rule weights are used to normalise fuzzy rules in a probabilistic sense. However, the semantics of using weights for fuzzy rules are critical.

\section{Conclusions}

Fuzzy classifiers - especially when combined with neural network learning - offer interpretable rules and often yield good results.

However, the impacts on the classification behaviour from simplifications of the rules can be grave. The occupation effect may in some situation be counter-intuitive. Pruned rules have the tendency to dominate the remaining rules. This should be kept in mind when pruning is performed. The adaptation of the fuzzy sets in an additional learning step can mostly compensate this. However, interpretability of the fuzzy sets might suffer.

\section{References}

[1] D. Gustafson, and W. Kessel, Fuzzy Clustering with a Fuzzy Covariance Matrix, In: Proc. IEEE CDC, pp. 761-766, San Diego, CA, 1979.

[2] F. Höppner, F. Klawonn, R. Kruse, and T. Runkler, Fuzzy Cluster Analysis, Wiley, Chichester, 1999.
[3] F. Klawonn, and E. Klement, Mathematical Analysis of Fuzzy Classifiers, In: X. Liu, P. Cohen, and M. Berthold (eds.), Advances in Intelligent Data Analysis, pp. 359-370, Springer, Berlin, 1997.

[4] F. Klawonn, and R. Kruse, Constructing a Fuzzy Controller from Data, Fuzzy Sets and Systems, 85, pp. 177-193, 1997.

[5] A. Klose, A. Nürnberger, and D. Nauck, Some Approaches to Improve the Interpretability of Neuro-Fuzzy Classifiers, In: Proc. 6th European Congress on Intelligent Techniques and Soft Computing (EUFIT'98), pp. 629-633, Aachen, 1998.

[6] R. Kruse, J. Gebhardt, and F. Klawonn, Foundations of Fuzzy Systems, Wiley, 1994.

[7] D. Nauck, F. Klawonn, and R. Kruse, Foundations of Neuro-Fuzzy Systems, Wiley, Chichester, 1997.

[8] D. Nauck, A. Nürnberger, and R. Kruse, NeuroFuzzy Classification, In: Advances in Data Science and Classification, Proc. of the 6th Conference of the International Federation of Classification Societies (IFCS-98), pp. 287-294, Springer-Verlag, Berlin, 1998.

[9] A. Nürnberger, C. Borgelt, and A. Klose, Improving Naive Bayes Classifiers Using Neuro-Fuzzy Learning, In: Proc. 6th International Conference on Neural Information Processing '99 (ICONIP'99), 6p., Perth, Australia, 1999.

[10] A. Nürnberger, A. Klose, and R. Kruse, Discussing Cluster Shapes of Fuzzy Classifiers, In: Proc. 18th International Conference of the North American Fuzzy Information Processing Society (NAFIPS'99), pp. 546-550, New York, 1999.

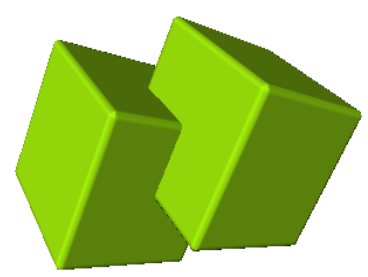

(a)

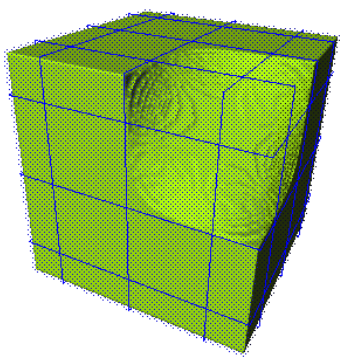

(e)

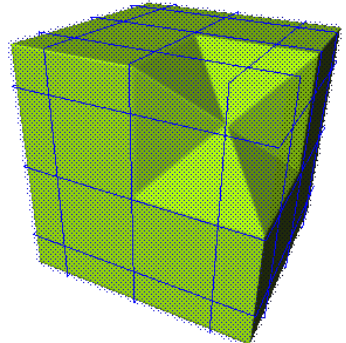

(b)

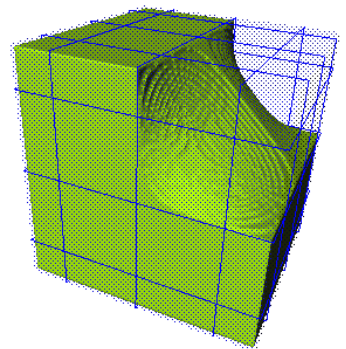

(f)

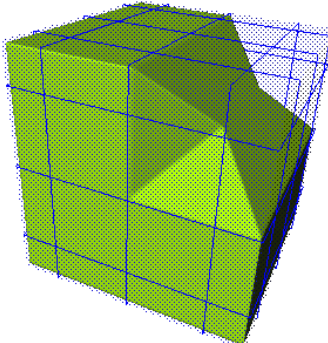

(c)

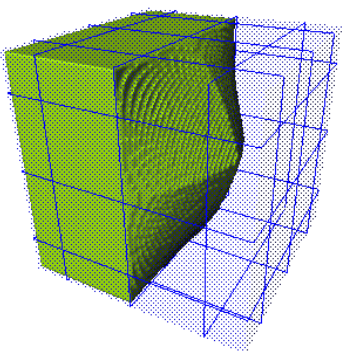

(g)

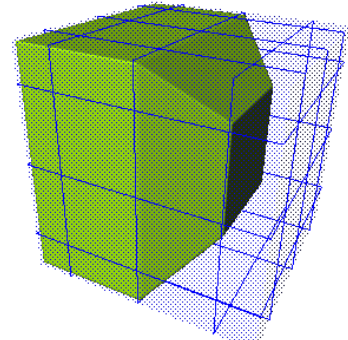

(d)

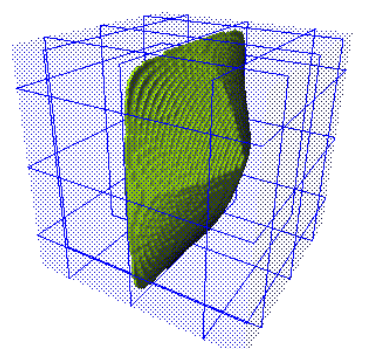

(h)

Figure 3. Pruned rule bases: two rules in 3D (a); one rule hidden, intersection surface visible for min (b) and prod (e); one and two antecedents pruned for $\min$ (c) and (d), and prod (f) and (g); introduced a third rule with two antecedents pruned (h) 